\title{
www.czasopisma.pan.pl \\ Modeling of Melting and Resolidification in Domain of Metal Film Subjected to a Laser Pulse
}

\author{
E. Majchrzak ${ }^{\mathrm{a} *}$, B. Mochnacki ${ }^{\mathrm{b}}$ \\ ${ }^{a}$ Silesian University of Technology, Konarskiego 18a, 44-100 Gliwice, Poland \\ ${ }^{\mathrm{b}}$ Higher School of Labour Safety Management, Bankowa 8, 40-007 Katowice Poland \\ *Corresponding author. E-mail address: ewa.majchrzak@polsl.pl
}

Received 18.06.2015; accepted in revised form 17.07.2015

\begin{abstract}
Thermal processes in domain of thin metal film subjected to a strong laser pulse are discussed. The heating of domain considered causes the melting and next (after the end of beam impact) the resolidification of metal superficial layer. The laser action (a time dependent belltype function) is taken into account by the introduction of internal heat source in the energy equation describing the heat transfer in domain of metal film. Taking into account the extremely short duration, extreme temperature gradients and very small geometrical dimensions of the domain considered, the mathematical model of the process is based on the dual phase lag equation supplemented by the suitable boundary-initial conditions. To model the phase transitions the artificial mushy zone is introduced. At the stage of numerical modeling the Control Volume Method is used. The examples of computations are also presented.
\end{abstract}

Keywords: Theoretical basis of foundry processes, Micro-scale heat transfer, Dual phase lag model, Melting and solidification, Numerical methods

\section{Introduction}

The base for the macroscopic model of alloys solidification or melting is the Fourier equation with an additional term (the source function) controlling the evolution of latent heat $[1,2,3]$. With the introduction of parameter called the substitute thermal capacity (e.g. [2]) the governing equation concerns the whole, conventionally homogeneous metal domain (one domain method). In the case of pure metal solidification the similar approach can be used, but the solidification point $T^{*}$ must be substituted by a certain temperature interval $\left[T^{*}-\Delta T, T^{*}+\Delta T\right]$. Next, for this interval the substitute thermal capacity should be defined. It turned out that the width of this interval is not critical for the results of numerical simulations [4]. In literature, as a rule, the macro models of solidification are discussed (e.g. [1 - 8]. In this paper the similar concept is applied, but the governing equation describing the thermal processes in domain of metal film corresponds to the dual phase lag equation. DPLE results from the generalized form of the Fourier law and it contains two lag times, in particular the relaxation and thermalization times $[9,10,11]$. Such a model describes well the heat transfer in micro-scale when the values of lag times are significant because of extremely short duration, extreme temperature gradients and small dimensions of the domain. The external action of laser beam is substituted by the introduction of internal volumetric heat source $[9,10]$ and then on the surface limiting the domain of metal film the adiabatic boundary conditions should be taken into account. Additionally, the initial temperature distribution and the initial heating rate are 
assumed to be known. The other approaches to the microscale heat transfer modelling are also used, e.g. [12, 13].

\section{Governing equations}

The following generalization of the Fourier law is introduced

$\mathbf{q}\left(r, z, t+\tau_{q}\right)=-\lambda \nabla T\left(r, z, t+\tau_{T}\right)$

where $\tau_{q}$ and $\tau_{T}$ are the phase lags (relaxation and thermalization times), while $\mathbf{q}$ is a heat flux, $\lambda$ is a thermal conductivity, $r, z$ are the geometrical co-ordinates (axially-symmetrical problem is considered), $t$ is a time.

Using the Taylor series expansions, the following first-order approximation of equation (1) is obtained

$\mathbf{q}(r, z, t)+\tau_{q} \frac{\partial \mathbf{q}(r, z, t)}{\partial t}=-\lambda\left[\nabla T(r, z, t)+\tau_{T} \frac{\partial \nabla T(r, z, t)}{\partial t}\right]$

Introducing this formula to the well known diffusion equation after the mathematical manipulations one has

$$
\begin{gathered}
c\left[\frac{\partial T(r, z, t)}{\partial t}+\tau_{q} \frac{\partial^{2} T(r, z, t)}{\partial t^{2}}\right]=\nabla[\lambda \nabla T(r, z, t)]+ \\
\tau_{T} \frac{\partial}{\partial t}\{\nabla[\lambda \nabla T(r, z, t)]\}+Q(r, z, t)+\tau_{q} \frac{\partial Q(r, z, t)}{\partial t}
\end{gathered}
$$

Here $c$ is a volumetric specific heat of material, $Q$ is the capacity of internal heat sources.

The mathematical formula determining the intensity of heat source $Q_{L}(r, z, t)$ resulting from the laser action is assumed in the form

$$
\begin{aligned}
& Q_{L}(r, z, t)=\sqrt{\frac{4 \ln 2}{\pi}}(1-R) \frac{I_{0}}{\delta t_{p}} \times \\
& \exp \left[-\frac{r^{2}}{r_{D}^{2}}-\frac{z}{\delta}-4 \ln 2 \frac{\left(t-2 t_{p}\right)^{2}}{t_{p}^{2}}\right]
\end{aligned}
$$

where $I_{0}\left[\mathrm{~J} / \mathrm{m}^{2}\right]$ is a laser intensity, $t_{p}[\mathrm{~s}]$ is a characteristic time of laser pulse, $\delta[\mathrm{m}]$ is an optical penetration depth, $R$ is a reflectivity of irradiated surface, $r_{D}[\mathrm{~m}]$ is a laser beam radius. The derivative of $Q_{L}$ with respect to time (equation (4)) can be found analytically. The next internal heat sources result from the phase changes

$$
Q_{S}(r, z, t)=L \frac{\partial f_{S}(r, z, t)}{\partial t}=L \frac{\mathrm{d} f_{S}(T)}{\mathrm{d} T} \frac{\partial T(r, z, t)}{\partial t}
$$

where $L$ is a volumetric latent heat, $f_{S}$ is a volumetric solid state fraction at the neighborhood of point considered.

We introduce the artificial mushy zone by 'stretching' of solidification point $T^{*}$ to a certain interval $\left[T^{*}-\Delta T, T^{*}+\Delta T\right]$ and for this interval the function $f_{S}$ is assumed in the linear form, this means.
$f_{S}(r, z, t)=\frac{T^{*}+\Delta T-T(r, z, t)}{2 \Delta T}$

One can see that for the border temperatures the function $f_{S}$ takes the values 0 and 1 . The source term connected with the evolution of latent heat takes a form

$$
\begin{aligned}
& Q_{S}(r, z, t)+\tau_{q} \frac{\partial Q_{S}(r, z, t)}{\partial t}=L \frac{\mathrm{d} f_{S}(T)}{\mathrm{d} T} \frac{\partial T(r, z, t)}{\partial t}+ \\
& \tau_{q} L\left\{\frac{\mathrm{d}^{2} f_{S}(T)}{\mathrm{d} T^{2}}\left[\frac{\partial T(r, z, t)}{\partial t}\right]^{2}+\frac{\mathrm{d} f_{S}(T)}{\mathrm{d} T} \frac{\partial^{2} T(r, z, t)}{\partial t^{2}}\right\}
\end{aligned}
$$

Taking into account that the second derivative of the function $f_{S}$ is equal to zero one finally obtains

$$
\begin{aligned}
& Q_{S}(r, z, t)+\tau_{q} \frac{\partial Q_{S}(r, z, t)}{\partial t}= \\
& L \frac{\mathrm{d} f_{S}(T)}{\mathrm{d} T}\left[\frac{\partial T(r, z, t)}{\partial t}+\tau_{q} \frac{\partial^{2} T(r, z, t)}{\partial t^{2}}\right]
\end{aligned}
$$

So, the energy equation (3) can be written in the form

$$
\begin{gathered}
C\left[\frac{\partial T(r, z, t)}{\partial t}+\tau_{q} \frac{\partial^{2} T(r, z, t)}{\partial t^{2}}\right]=\nabla[\lambda \nabla T(r, z, t)]+ \\
\tau_{T} \frac{\partial}{\partial t}\{\nabla[\lambda \nabla T(r, z, t)]\}+Q_{L}(r, z, t)+\tau_{q} \frac{\partial Q_{L}(r, z, t)}{\partial t}
\end{gathered}
$$

where $C$ is a substitute thermal capacity of mushy zone

$$
C=c_{M}-L \frac{\mathrm{d} f_{S}(T)}{\mathrm{d} T}=c_{M}+\frac{L}{2 \Delta T}, \quad T \in\left[T^{*}-\Delta T, T^{*}+\Delta T\right]
$$

where $c_{M}$ is the volumetric specific heat of this sub-region. Summing up, the substitute thermal capacity is defined as follows

$C(T)=\left\{\begin{array}{lc}c_{L} & T>T^{*}+\Delta T \\ c_{M}+\frac{L}{2 \Delta T} & T^{*}-\Delta T \leq T \leq T^{*}+\Delta T \\ c_{S} & T<T^{*}-\Delta T\end{array}\right.$

where $c_{L}, c_{S}$ are the volumetric specific heats of liquid and solid state, while (for example) $c_{M}$ is the arithmetic mean of $c_{L}$ and $c_{S}$. One can see that the equation (9) describes the thermal processes proceeding in whole, conventionally homogeneous metal domain. The adiabatic boundary condition given on the external surface of the system is of the form [14] 
$-\lambda\left[\mathbf{n} \cdot \nabla T(r, z, t)+\tau_{T} \frac{\partial[\mathbf{n} \cdot \nabla T(r, z, t)]}{\partial t}\right]=0$

where $\mathbf{n} \cdot \nabla T(r, z, t)$ is the temperature derivative in the normal direction.

The initial conditions (the initial temperature of domain and the initial heating rate) are also given

$t=0: \quad T(r, z, 0)=T_{0},\left.\quad \frac{\partial T(r, z, t)}{\partial t}\right|_{t=0}=0$

where $T_{0}$ is the constant initial temperature.

\section{Control Volume Method}

At the stage of numerical computations the Control Volume Mehod (CVM) in the version proposed by Mochnacki and Ciesielski [14] is applied. In the cited article one can find the comprehensive information concerning the method application in the case of hyperbolic equations. As is well known, The CVM algorithm allows one to find the transient temperature field at the set of nodes corresponding to the central points of control volumes. The thermal capacities are concentrated at the nodes representing elements, while the thermal resistances are concentrated on the sectors joining the nodes. The nodal temperatures can be found on the basis of energy balances for the successive volumes. The energy balances corresponding to heat exchange between the analyzed control volume and adjoining control volumes results from the integration of equation (9) with respect to volume and time. In Figure 1 the cylindrical, axially symmetrical domain is shown.

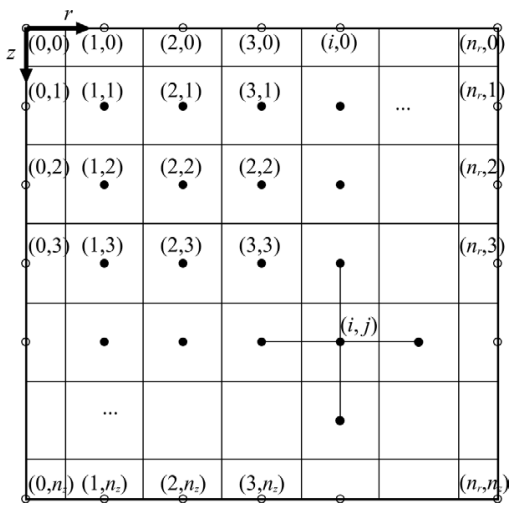

Fig. 1. The discretization of domain

To construct the CVM equations, the successive volumes $\Delta V_{i, j}$ and the values of surfaces limiting $\Delta V_{i, j}$ must be known, and they can be found on the basis of the simple geometrical considerations (annular elements).

Without going into the detais the CVM approximation of the left hand side of equation (9) is the following
$\mathbf{L}=C_{i, j}^{f-1}\left(\frac{T_{i, j}^{f}-T_{i, j}^{f-1}}{\Delta t}+\tau_{q} \frac{T_{i, j}^{f}-2 T_{i, j}^{f-1}+T_{i, j}^{f-2}}{(\Delta t)^{2}}\right) \Delta V_{i, j}(13)$

where $\Delta t$ is a time step. Above formula shows that in the case of hyperbolic equations three-level CVM approximation must be used, in other words, in order to determine the temperature $T_{i, j}^{f}$ (time level $f$ ) the nodal temperatures corresponding to time levels $f-1$ and $f-2$ must be known. Taking into account the CVM approximation of the right hand side of equation (9), finally one obtains the linear algebraic equations from which the nodal temperatures for time $t^{f}$ can be found. Becduse the explicit scheme has been used, therefore the stability conditions should be fulfilled.

\section{Examples of computations}

Numerical simulation of a thermal process subjected to the short-pulse laser heating has been done for the cylindrical domain with dimensions $Z=100 \cdot 10^{-9} \mathrm{~m}, R=100 \cdot 10^{-9} \mathrm{~m}$ - Figure 2 [14].

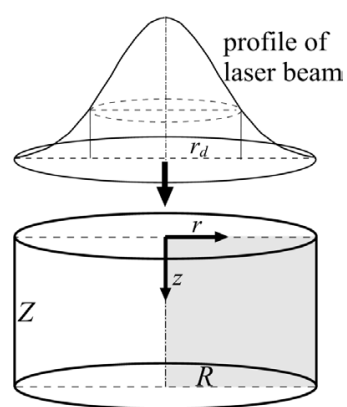

Fig. 2. Micro-domain considered

Thermophysical parameters of material (chromium) are equal to $\lambda=93 \quad \mathrm{~W} /(\mathrm{mK}), \quad c=3.2148 \cdot 10^{6} \quad \mathrm{~J} /\left(\mathrm{m}^{3} \mathrm{~K}\right), \quad L=2904 \cdot 10^{6} \mathrm{~J} / \mathrm{m}^{3}$, solidification point $T^{*}=1857^{\circ} \mathrm{C}, \Delta T=5 \mathrm{~K}, \tau_{q}=0.136 \cdot 10^{-12} \mathrm{~s}$, $\tau_{T}=7.86 \cdot 10^{-12} \mathrm{~s}$ [15]. The parameters of the Gaussian laser pulse are following: $r_{d}=50 \cdot 10^{-9} \mathrm{~m}, \quad I_{0}=10 \mathrm{~kW} / \mathrm{m}^{2}, \quad R_{f}=0.93$, $\delta=15.3 \cdot 10^{-12} \mathrm{~m}, t_{p}=50 \cdot 10^{-12} \mathrm{~s}$. The initial temperature of the metal is $T_{0}=20^{\circ} \mathrm{C}$. The mesh steps used in this example: $\Delta z=2 \cdot 10^{-9} \mathrm{~m}$, $\Delta r=2 \cdot 10^{-9} \mathrm{~m}$ and the time step $\Delta \mathrm{t}=0.5 \cdot 10^{-15} \mathrm{~s}$. The results of computations are shown in Figures 3 and 4. Figure 3 illustrates the temperarure distribution for time $100 \cdot 10^{-12} \mathrm{~s}$. In Figure 4 the temperature history at the nodes $\mathrm{A}(0,0)$ and $\mathrm{B}\left(0,49 \cdot 10^{-9} \mathrm{~m}\right)$ is presented.

\section{Final remarks}

The authors solved the problem of pure metal melting and resolidification by the introduction of artificial mushy zone to the mathematical model od the process. The assumption of the function describing the volumetric fraction of solid in the linear 
form allowed one to obtain the DPL equation in the form containing the well known parameter called a substitute thermal capacity. At the stage of numerical solution of the example presented in the Chapter 4 the value $\Delta T=5 \mathrm{~K}$ has been assumed, but the testing computations showed that the interval $\Delta T$ width can be changed. The observation of numerical results confirmed the very small changes of temperature close to the solidification point. Unfortunately, this effect is not clearly visible in Figure 4 (the short duration and the narrow interval $2 \Delta T$ ).

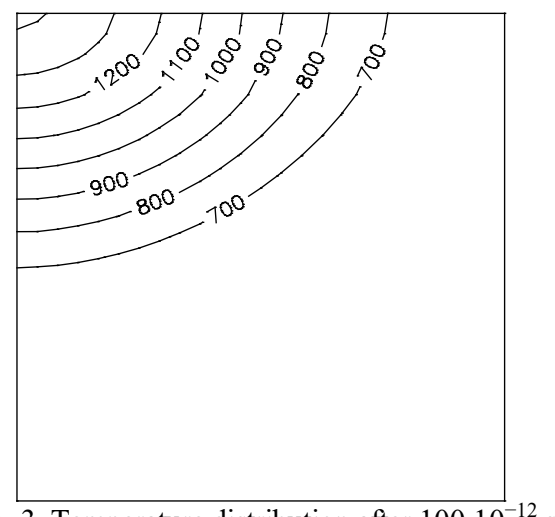

Fig. 3. Temperature distribution after $100 \cdot 10^{-12} \mathrm{~s}$

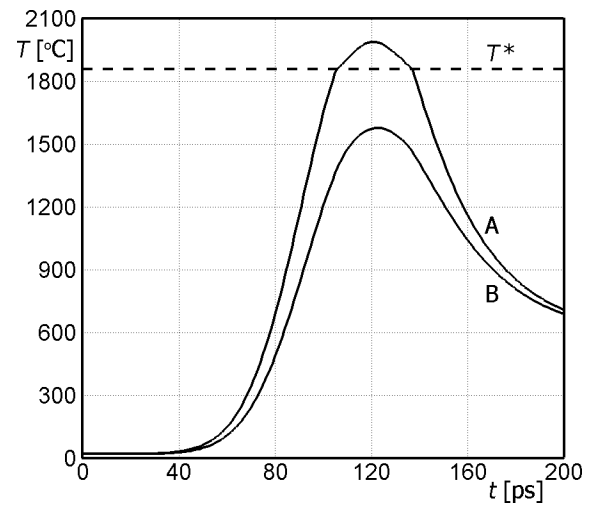

Fig. 4. Temperature history at the nodes $\mathrm{A}$ and $\mathrm{B}$

\section{Acknowledgement}

The paper is a part of Project 2012/05/B/ST8/01477 sponsored by National Science Centre.

\section{References}

[1] Mochnacki, B. \& Majchrzak, E. (2010). Numerical modeling of casting solidification using generalized finite difference method, Materials Science Forum, 638-642, 2676-2681.

[2] Mochnacki, B. (2012). Definition of alloy substitute thermal capacity using the simple macrosegregation models, Archives of Foundry Engineering, 19, 4, 113-116.

[3] Mochnacki, B. (2011). Computational simulations and applications, Numerical modeling of solidification process (Chapter 24), Ed. Jianping Zhu, INTECH, 513-542.

[4] Szopa, R. (2015). Numerical modeling of pure metal solidification using the one domain approach, Journal of Applied Mathematics and Computational Mechanics, 14, 3, 28-34.

[5] Bondarenko, V.I., Bilousov, V.V., Nedopekin, F.V. \& Shalapko, J.I. (2015). The mathematical model of hydrodynamics and heat and mass transfer at formation of steel ingots and castings, Archives of Foundry Engineering, $15,1,13-16$.

[6] Ivanova, A.A. (2012). Calculation of phase change boundary position in continuous casting, Archives of Foundry Engineering, 13, 4, 57-62.

[7] Mochnacki, B. \& Majchrzak, E. (2007). Identification of macro and micro parameters in solidification model, Bulletin of the Polish Academy of Sciences: Technical Sciences, 55,1, 107-113.

[8] Majchrzak, E. \& Mochnacki, B. (2007). Identification of thermal properties of the system casting-mould, Material Science Forum, 539-543, 2491-2496.

[9] Chen, J.K. \& Beraun, J.E. (2001), Numerical study of ultrashort laser pulse interactions with metal films, Numerical Heat Transfer, Part A, 40, 1-20.

[10] Zhang, Z.N. (2007). Nano/microscale heat transfer, McGraw-Hill, New York.

[11] Chen, G., Borca-Tasciuc, D., Yang, R.G. (2004). Nanoscale heat Transfer, Encyclopedia of NanoScience and Nanotechnology, 7, 429-459.

[12] Majchrzak E., Dziatkiewicz J. (2012) Numerical modeling of melting process of thin metal film subjected to the short laser pulse, Archives of Foundry Engineering, 12, 4, 105-108.

[13] Piasecka-Belkhayat, A., Korczak, A. (2014). Modeling of transient heat transport in one-dimensional crystalline solids using the interval lattice Boltzman method, in: $\mathrm{T}$. Lodygowski, J.Rakowski, P.Litewka, Recent Advances in Computational Mechanics, CRC Press, 363-367.

[14] Mochnacki, B., Ciesielski, M. (2015). Micro-scale heat transfer. Algorithm basing on the Control Volume Method and the identification of relaxation and thermalization times using the search method, Computer Methods in Materials Science (in print).

[15] Tang, D.W., Araki, N. (1999). Wavy, wavelike, diffusive thermal responses of finite rigid slabs to high-speed heating of laser-pulses. International Journal of Heat and Mass Transfer, 42, 855-860. 